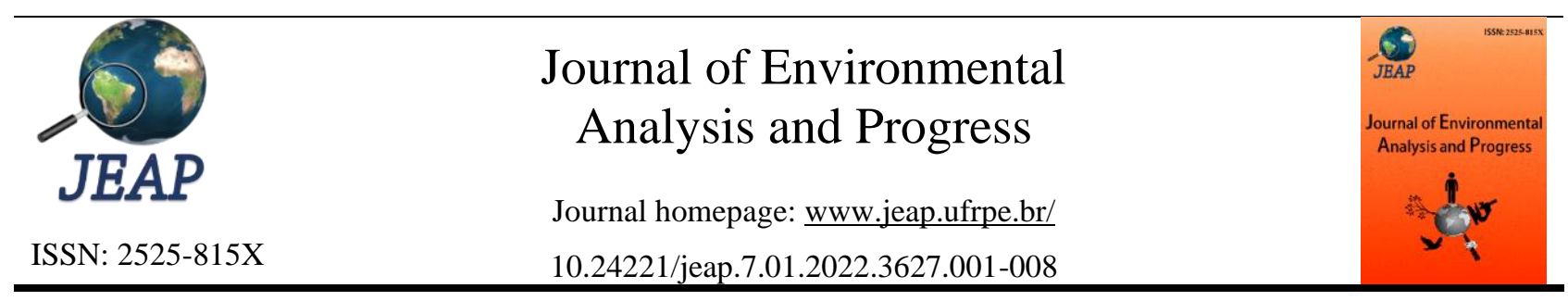

\title{
Reconstrução da paleoprodutividade e sua relação com as contribuições continentais no estuário de Macuse, Centro de Moçambique
}

\section{Reconstruction of paleoproductivity and its relationship with continental contributions in the Macuse estuary, Central Mozambique}

\author{
Adelina António Langa ${ }^{\mathrm{a}}$, Hélder Arlindo Machaieie ${ }^{\mathrm{b}}$ \\ ${ }^{a}$ Escola Superior de Ciências Marinhas e Costeiras, Universidade Eduardo Mondlane, Departamento de Geologia \\ Marinha, Rua do Chuabo Dembe, Quelimane, Moçambique. CEP: 08-128. E-mail: adelinalanga.al@ gmail.som. \\ b Escola Superior de Ciências Marinhas e Costeiras, Universidade Eduardo Mondlane, Rua do Chuabo Dembe, \\ Quelimane, Moçambique. CEP: 08-128. E-mail: machaielder@gmail.com.
}

\begin{abstract}
A R T I C L E I N F O
Recebido 16 Jun 2020

Aceito 22 Dez 2021

Publicado 17 Jan 2022

A B S T R A C T

The reconstruction of paleoproductivity allows understanding of marine life and the associated processes in geological time scale. The Macuse estuary is an environment with high biogeochemical reactivity and is particularly sensitive to local, regional, and global climatic variations. The study aimed to reconstruct paleoproductivity and establish its relationship with continental contributions in the Macuse estuary. An analysis was carried out on a $90 \mathrm{~cm}$ deep core, subdivided into nine sediment subsamples of $10 \mathrm{~cm}$ of length each. It was necessary to weigh $20 \mathrm{~g}$ of dry sediment and insert it in an X-ray fluorescence spectrometer by EDX-7000 energy dispersion for quantitative analysis of the elements $\mathrm{Al}, \mathrm{Fe}, \mathrm{Ti}, \mathrm{Si}, \mathrm{K}$, and $\mathrm{Ca}$. Paleoproductivity estimates were based on the $\mathrm{Si} / \mathrm{Ti}, \mathrm{Ca} / \mathrm{Ti}$, and $\mathrm{K} / \mathrm{Ti}$ ratios; the continental contributions were inferred through the $\mathrm{Al} / \mathrm{Ca}, \mathrm{Ti} / \mathrm{Ca}$, and $\mathrm{Fe} / \mathrm{Ca}$ ratios. The profiles of paleoproductivity indicators were characterized by high values at $10 \mathrm{~cm}, 70 \mathrm{~cm}$, and $90 \mathrm{~cm}$ depths. Considering the continental contribution, the profiles were characterized by low values, at depths of $70 \mathrm{~cm}$ to $90 \mathrm{~cm}$, and high values at $50 \mathrm{~cm}$. The increased productivity events in the Macuse estuary coincided with a low continental contribution, suggesting that the continental contribution did not enhance paleoproductivity. However, this relationship may not be consistent since the correlation between the continental contribution and the sediment grain size showed that it controls the concentration of continental contribution indicators.
\end{abstract}

Keywords: Reconstruction of paleoproductivity, continental contribution, biogeochemical elements, grain size.

\footnotetext{
R E S U M O

A reconstrução da paleoprodutividade permite a compreensão da vida marinha e dos processos associados na escala de tempo geológico. O estuário de Macuse é um ambiente com alta reactividade biogeoquímica e particularmente sensível a variações climáticas locais, regionais e globais. $O$ estudo objetivou reconstruir a paleoprodutividade e estabelecer sua relação com as contribuições continentais no estuário de Macuse. Foi realizada uma análise de nove amostras de sedimentos correspondentes a um testemunhador de $90 \mathrm{~cm}$ de profundidade, com uma subamostragem de $10 \mathrm{~cm}$. Foi necessário pesar $20 \mathrm{~g}$ de sedimento seco e inserir no espectrômetro de fluorescência de raios X por dispersão de energia EDX-7000 para a análise quantitativa dos elementos $\mathrm{Al}, \mathrm{Fe}, \mathrm{Ti}, \mathrm{Si}, \mathrm{K}$ e $\mathrm{Ca}$. As estimativas de paleoprodutividade foram baseadas nas relações $\mathrm{Si} / \mathrm{Ti}, \mathrm{Ca} / \mathrm{Ti}$ e $\mathrm{K} / \mathrm{Ti}$ e as contribuições continentais foram $\mathrm{Al} / \mathrm{Ca}, \mathrm{Ti} / \mathrm{Ca}$ e $\mathrm{Fe} / \mathrm{Ca}$. Os indicadores de paleoprodutividade apresentam perfis caracterizados por altos valores nas profundidades de $10 \mathrm{~cm}, 70 \mathrm{~cm}$ e $90 \mathrm{~cm}$; para a contribuição continental, os perfis foram caracterizados por valores baixos, nas profundidades de $70 \mathrm{~cm}$ a $90 \mathrm{~cm}$, e altos valores aos $50 \mathrm{~cm}$. Os eventos de alta produtividade no estuário de Macuse
} 
coincidiram com uma baixa contribuição continental, sugerindo que a paleoprodutividade não foi estimulada pela contribuição continental. Contudo, essa relação pode não ser confiável, uma vez que a correlação entre a contribuição continental e a granulometria de sedimento mostrou que a concentração dos indicadores de contribuição continental é controlada pela granulometria do sedimento.

Palavras-chave: Reconstrução da paleoprodutividade, contribuição continental, elementos biogeoquímicos, granulometria de sedimento.

\section{Introdução}

Nos últimos anos, estudos paleoceanográficos têm ganho mais espaço no que se refere à área das geociências. Por via da reconstrução paleoambiental, paleoclimática e da paleoprodutividade é possível observar mudança nas condições climáticas e na dinâmica dos oceanos passados e assim perspectivar futuras alterações no meio marinho.

A paleoceanografia utiliza parâmetros físicos, químicos e biológicos encontrados em registos sedimentares marinhos, na elaboração de relações entre a variação de padrões apresentados por parâmetros oceanográficos ao longo do tempo e as respostas do sistema oceano-atmosfera (Souza, 2016). Os estudos paleoceanográficos são comummente realizados em ambientes marinhos e estuarinos.

Segundo Veronez Junior (2009), ambientes estuarinos são preferenciais para estudos de reconstituição de paleoprodutividade, por tratarse de locais de condições com alta produtividade biológica, pois, há muita produção de matéria orgânica e entrada de nutrientes provenientes dos ambientes adjacentes (rios e região costeira).

A reconstituição da paleoprodutividade desempenha um papel importante no controle do fluxo de carbono entre o meio marinho e/ou estuarino e a atmosfera, o registo de suas flutuações pode, portanto, influenciar o clima, alterando as concentrações de $\mathrm{CO}_{2}$ na atmosfera (Andrade, 2011). Para além de contribuir para a compreensão do ciclo de carbono, estudos relacionados à paleoprodutividade são importantes para o desenvolvimento de inferências em áreas como a biogeoquímica, padrões climáticos de um determinado ambiente (Martins et al., 2006).

$\mathrm{O}$ estudo das condições ambientais tem sido baseado em registos obtidos nos sedimentos marinhos, pois são ambientes estáveis em termos físico e químico (Martins et al., 2007).

Em ambientes costeiros como estuários são comummente preservados sequências de sedimentos do Holoceno, oferecendo a oportunidade de investigar o clima do passado e mudanças no nível do mar (Lamb et al., 2006), porém a precisão das reconstruções de condições ambientais depende da qualidade de banco de dados, entretanto os sedimentos recuperados do fundo dos estuários documentam as variações ambientais e climáticas ocorridas na região onde os materiais se depositaram, pois os depósitos são contínuos e a designação de idade é geralmente mais confiável.

De acordo com Santos (2010) a informação documentada pelo registo sedimentar é passível de ser estudada utilizando indicadores (proxies) ou marcadores (tracers) das condições ambientais e/ou climáticas.

Destaca-se o uso de elementos maiores e traços como indicadores de produtividade marinha: MO (matéria orgânica), COT (carbono orgânico total), $\mathrm{P}$ (fósforo), $\mathrm{Si}$ (silício), $\mathrm{Ca}$ (cálcio), $\mathrm{CaCO}_{3}$ (carbonato de cálcio), Ba (bário), e razões $\mathrm{Al} / \mathrm{Ti}$ (alumínio/titânio), $\mathrm{Si} / \mathrm{Ti}$ (silício/titânio), $\mathrm{K} / \mathrm{Ti}$ (potássio/titânio), $\mathrm{Ca} / \mathrm{Ti}$ (cálcio/titânio) entre outros. Indicadores de contribuição continental razões $\mathrm{C} / \mathrm{N}$ (carbono/nitrogénio), $\mathrm{Fe} / \mathrm{Ca}$ (ferro/cálcio), $\quad \mathrm{Ti} / \mathrm{Ca} \quad$ (titânio/cálcio), $\quad \mathrm{Al} / \mathrm{Ca}$ (alumínio/cálcio).

Dos indicadores acima apresentados existem aqueles que apresentam maior estabilidade e preservação com menos influência dos factores externos o caso de $\mathrm{Si} / \mathrm{Ti}, \mathrm{Ca} / \mathrm{Ti}$ e $\mathrm{K} / \mathrm{Ti}$ para a paleoprodutividade (Piñeiro, 2013) e Al/Ca, Fe/Ca e Ti/Ca para as contribuições continentais (Souza, 2016).

Atestando a importância e a vantagem destes indicadores em reconstruir paleoambientes, neste estudo foram usados os indicadores acima citados para a reconstrução das condições paleoambientais. Neste contexto, o estudo tem como objetivo principal reconstruir a paleoprodutividade e sua relação com as contribuições continentais no estuário de Macuse. Pretende-se, também, com esta pesquisa despertar o interesse dos cientistas sobre as mudanças climáticas, a ciclagem biogeoquímica dos elementos e a frequência dos processos envolvidos nas variações em diversas escalas espaço-temporal.

\section{Material e Métodos \\ Caracterização da área de estudo \\ $\mathrm{O}$ estuário de Macuse localiza-se na} Província da Zambézia, no centro de Moçambique, na região leste da África Austral. Localiza-se administrativamente no Distrito de Namacurra, entre as coordenadas geográficas: $17^{\circ} 48^{\prime} 1,43$ "S de 
latitude e $37^{\circ} 04^{\prime} 00$ "E de longitude. Limita-se a leste pelo Oceano Índico, a Norte e Oeste pelo Posto Distrital de Namacurra Sede e a Sul pelo Distrito de Nicoadala (Figura 1).

$\mathrm{O}$ rio principal é o Namacurra, apresenta um comprimento de cerca de $30 \mathrm{~km}$ da nascente até a desembocadura com o oceano Índico. É caracterizado por uma rede de pequenos afluentes que desaguam no canal principal (Miguel et al., 2017).
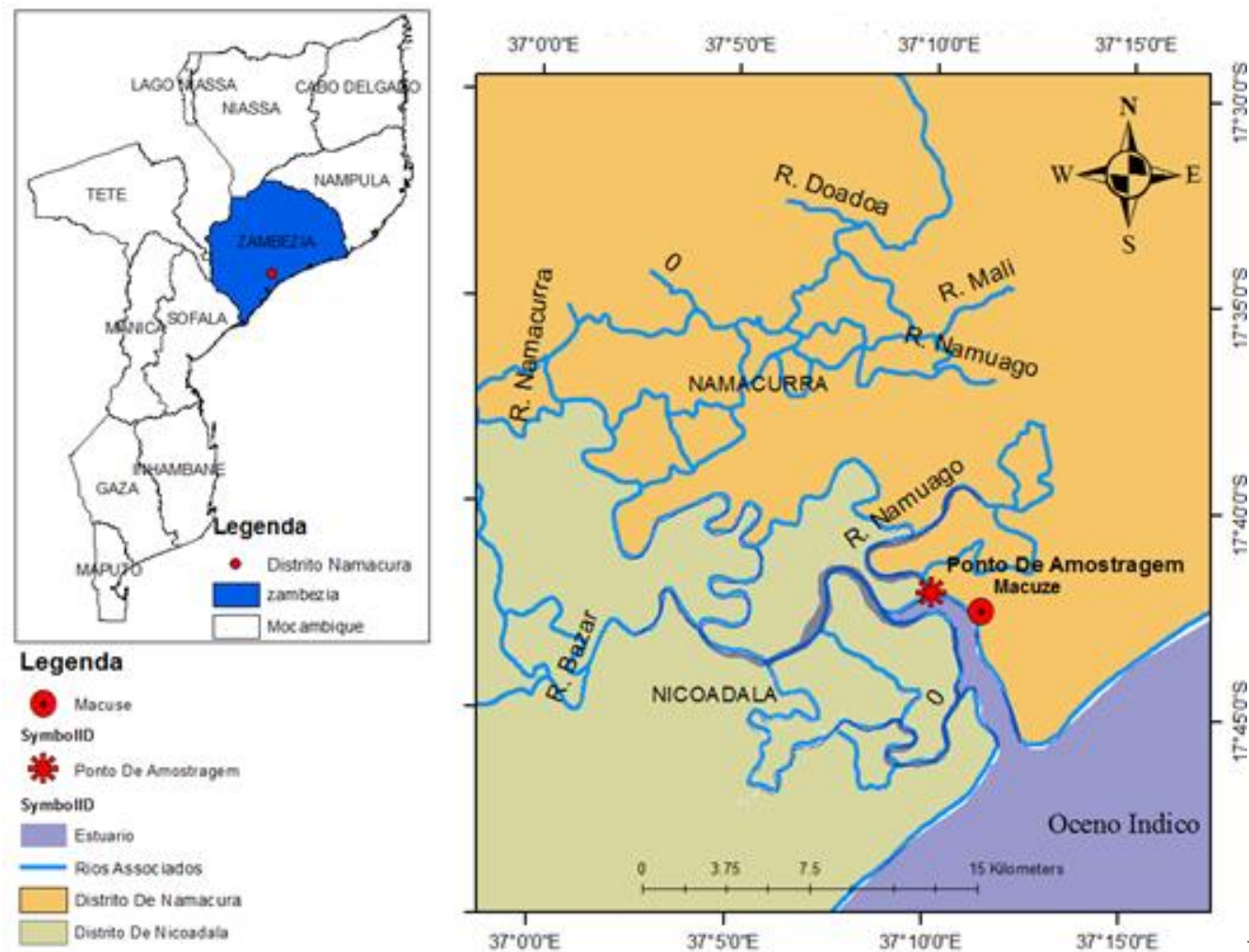

da área de estudo em Macuse, Zambézia, Moçambique. Fonte: Langa et al. (2019).

\section{Processo de amostragem}

Uma visita ao campo foi feita no dia 05Jul-2019 até o estuário de Macuse, com o intuito de coletar amostras de sedimentos do fundo. Para tal, construiu-se um (01) testemunhador (core), usando tubos PVC de $75 \mathrm{~mm}$ de diâmetro e $1.5 \mathrm{~m}$ de comprimento, fios de aço, parafusos e chapa de zinco (Figura 2A). Assim construído o testemunho, seguiu-se a coleta das amostras, verticalmente, para estabelecer a preservação da estratigrafia da coluna sedimentar (Figura 2B). As amostras foram coletadas em um período de maré de sizígia, particularmente na baixa-mar (pico mínimo da vazante), pelas $12 \mathrm{~h} 00 \mathrm{~min}$, para fácil acesso do estuário. Um GPS (sistema de posicionamento geográfico) foi usado para referenciar as coordenadas geográficas do ponto de amostragem: Latitude: $17^{\circ} 42^{\prime} 1.22^{\prime \prime S}$ e Longitude: $37^{\circ} 10^{\prime} 5.18^{\prime \prime} \mathrm{E}$.

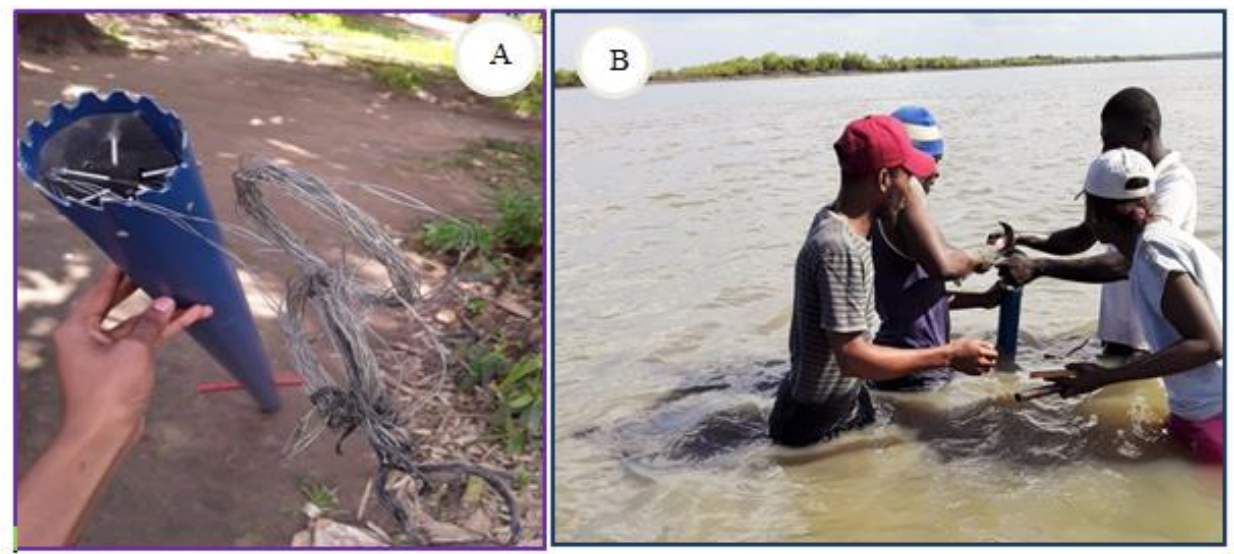

Figura 2. A. Testemunhador usado para a coleta dos sedimentos do fundo; B. quatro pessoas retirando sedimentos do fundo. Fonte: Langa et al. (2019). 


\section{Tratamento das amostras}

O testemunhador (core) foi cortado longitudinalmente em duas partes iguais, foi medido usando fita métrica a porção de sedimentos que era de, aproximadamente, $90 \mathrm{~cm}$ de profundidade; em seguida foram feitas subamostragens de $10 \mathrm{~cm}$. As amostras de sedimentos foram armazenadas em sacos plásticos devidamente codificadas, deixadas à temperatura ambiente e levadas ao laboratório de química da Faculdade de Ciências da Universidade Eduardo Mondlane. Chegando ao laboratório, as amostras foram deixadas secar a uma temperatura de $110^{\circ} \mathrm{C}$ durante 6 horas na estufa; posteriormente, as mesmas foram trituradas ou desagregadas usando um almofariz.

\section{Análise das amostras}

Para a determinação das concentrações dos elementos maiores e menores $\mathrm{Fe}, \mathrm{Ti}, \mathrm{Al}, \mathrm{Si}, \mathrm{Ca}$ e K recorreu-se ao método descrito por (Blanchet et al., 2007) no qual consiste em varrimento de fluorescência de raios-X. Foi necessário pesar $20 \mathrm{~g}$ de sedimentos seco, foram inseridas em cápsulas isoladas e pulverizados com o moinho de bolas antes da análise direta ao espectrômetro de fluorescência de raios $\mathrm{X}$ por dispersão de energia (EDXRF).

O espectrômetro de fluorescência de raios $\mathrm{X}$ por dispersão de energia EDX-7000 mede a energia $(\mathrm{keV})$ e a intensidade dos raios $\mathrm{X}$ fluorescentes gerados para determinar o tipo e o conteúdo dos elementos que compõem uma amostra.

É uma técnica não destrutiva, que permite a análise relativamente rápida e com elevada resolução. Cada espectro de energia é transformado por um processo de convolução, assistida por um computador, em conteúdos relativos expressos em contagens por segundo. O tempo de leitura para cada amostra foi de, aproximadamente, 5 minutos.

\section{Tratamento de dados}

Os dados da concentração total dos elementos maiores e menores ( $\mathrm{Fe}, \mathrm{Ti}, \mathrm{Al}, \mathrm{Si}, \mathrm{Ca}$ e $\mathrm{K})$ no estuário de Macuse foram lançados no Excel 2013 para o devido processamento. Foram calculadas as razões entre os elementos $\mathrm{Fe} / \mathrm{Ca}$, $\mathrm{Ti} / \mathrm{Ca}, \mathrm{Al} / \mathrm{Ca}, \mathrm{Si} / \mathrm{Ti}, \mathrm{Ca} / \mathrm{Ti}$ e K/Ti. Para a descrição das flutuações de contribuições (entradas) continentais foram usadas as razões elementares de $\mathrm{Fe} / \mathrm{Ca}, \mathrm{Ti} / \mathrm{Ca}$ e $\mathrm{Al} / \mathrm{Ca}$. As razões elementares $\mathrm{Si} / \mathrm{Ti}$, $\mathrm{Ca} / \mathrm{Ti}$ e $\mathrm{K} / \mathrm{Ti}$ como indicadores de produtividade no estuário (Piñeiro, 2013).

Para relacionar a variação (flutuações) das contribuições (entradas) continentais com a paleoprodutividade os dados $(\mathrm{Fe} / \mathrm{Ca}, \mathrm{Al} / \mathrm{Ca}, \mathrm{Si} / \mathrm{Ti}$ e $\mathrm{K} / \mathrm{Ti}$ ) foram efetuadas análises estatísticas, com correlação de Pearson, utilizando o programa computacional software past3. Esta correlação de Pearson é utilizada na descrição da associação entre variáveis independentes, não implicando em dependência entre as variáveis, nem relação de causa-e-efeito (Nagai, 2009), aplicando a Equação 1.

$\boldsymbol{r}=\frac{\sum\left(\mathbf{x}-\mathbf{x}^{\circ}\right) *\left(\mathbf{y}-\mathbf{y}^{\circ}\right)}{(\mathbf{n}-\mathbf{1}) * \mathbf{S} \mathbf{x} * \mathbf{S y}}$

Eq.(01)

onde: $\boldsymbol{x}=$ primeira variável; $\boldsymbol{y}=$ segunda variável; $\boldsymbol{x}^{\circ}=$ Média do conjunto de dados da primeira variável; $\boldsymbol{y}^{\circ}=$ Média do conjunto de dados da segunda variável; $\boldsymbol{S} \boldsymbol{x}=$ Desvio padrão do conjunto de dados da primeira variável e $\boldsymbol{S} \boldsymbol{y}=$ Desvio padrão do conjunto de dados da segunda variável.

\section{Resultados}

Os gráficos ilustrados na Figura 3 são correspondentes às flutuações das contribuições continentais para as razões elementares $\mathrm{Al} / \mathrm{Ca}$, $\mathrm{Ti} / \mathrm{Ca}$ e $\mathrm{Fe} / \mathrm{Ca}$, em percentagem (\%), ao longo da profundidade no ponto de amostragem. Os valores da razão $\mathrm{Al} / \mathrm{Ca}$ variam entre $2,32 \%$ e $7,47 \%$, da razão Ti/Ca variam entre $0,35 \%$ e $1,07 \%$ e para a razão $\mathrm{Fe} / \mathrm{Ca}$ variam entre $4,15 \%$ e $15,70 \%$.

Todos os gráficos apresentam perfis com o mesmo padrão de flutuações das contribuições continentais, aos $20 \mathrm{~cm}$ de profundidade observase um pequeno aumento das entradas continentais; os valores máximos são verificados na profundidade média aos $50 \mathrm{~cm}$ e os valores diminuem, gradualmente, até o pico mínimo aos 90 $\mathrm{cm}$ de profundidade, isto é, na base do testemunho. 


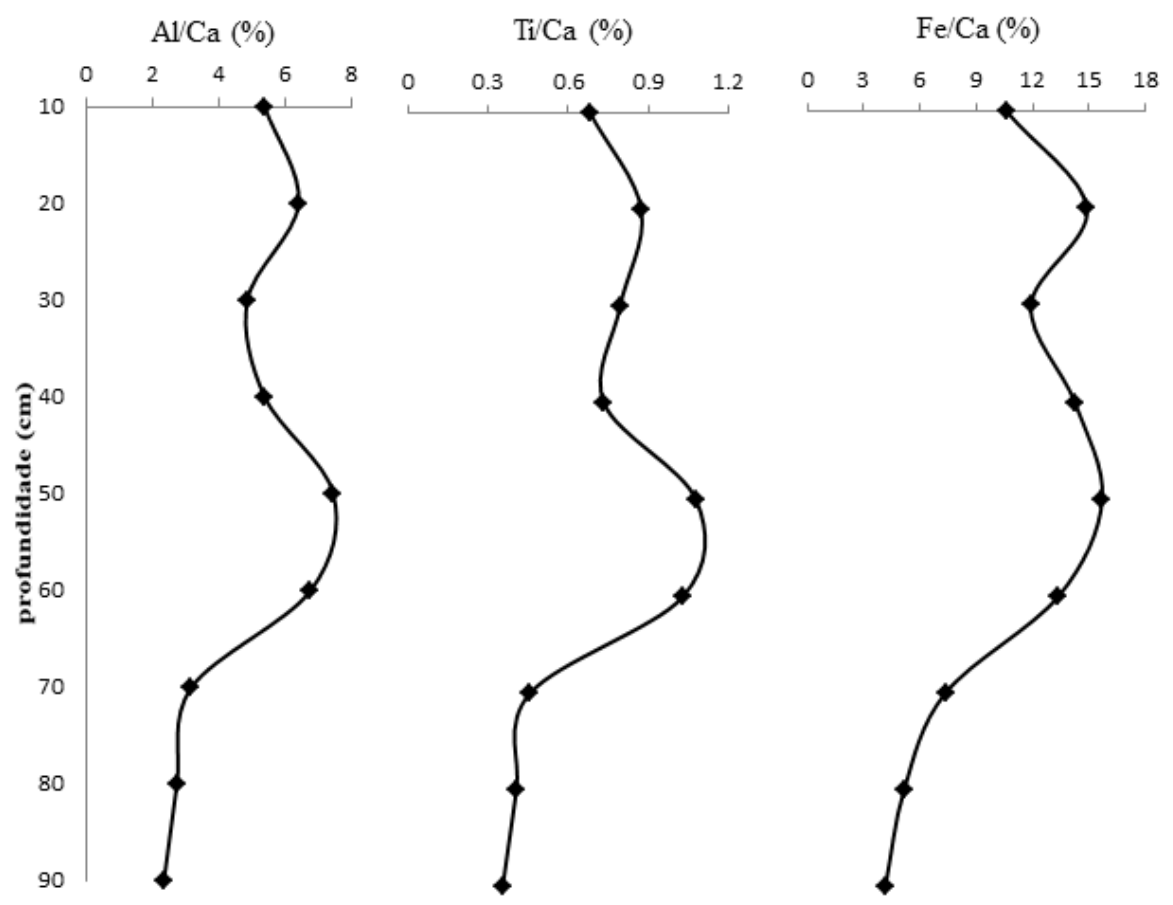

Figura 3. Perfis verticais dos indicadores das contribuições (entradas) continentais às razões elementares (Al/Ca, Ti/Ca e Fe/Ca). Fonte: Langa et al. (2019).

A Figura 4 ilustra os gráficos que correspondem as flutuações da paleoprodutividade as razões elementares de $\mathrm{Si} / \mathrm{Ti}, \mathrm{Ca} / \mathrm{Ti}$ e $\mathrm{K} / \mathrm{Ti}$ em percentagem $(\%)$ ao longo da profundidade no ponto de amostragem.

Os valores da razão $\mathrm{Si} / \mathrm{Ti}$ variam entre $17,47 \%$ e $24,44 \%$, para a razão $\mathrm{Ca} / \mathrm{Ti}$ variam entre $0,92 \%$ e $2,82 \%$ e para a razão $\mathrm{K} / \mathrm{Ti}$ variam entre $3,19 \%$ e $4,50 \%$.

$\mathrm{Si} / \mathrm{Ti}(\%)$

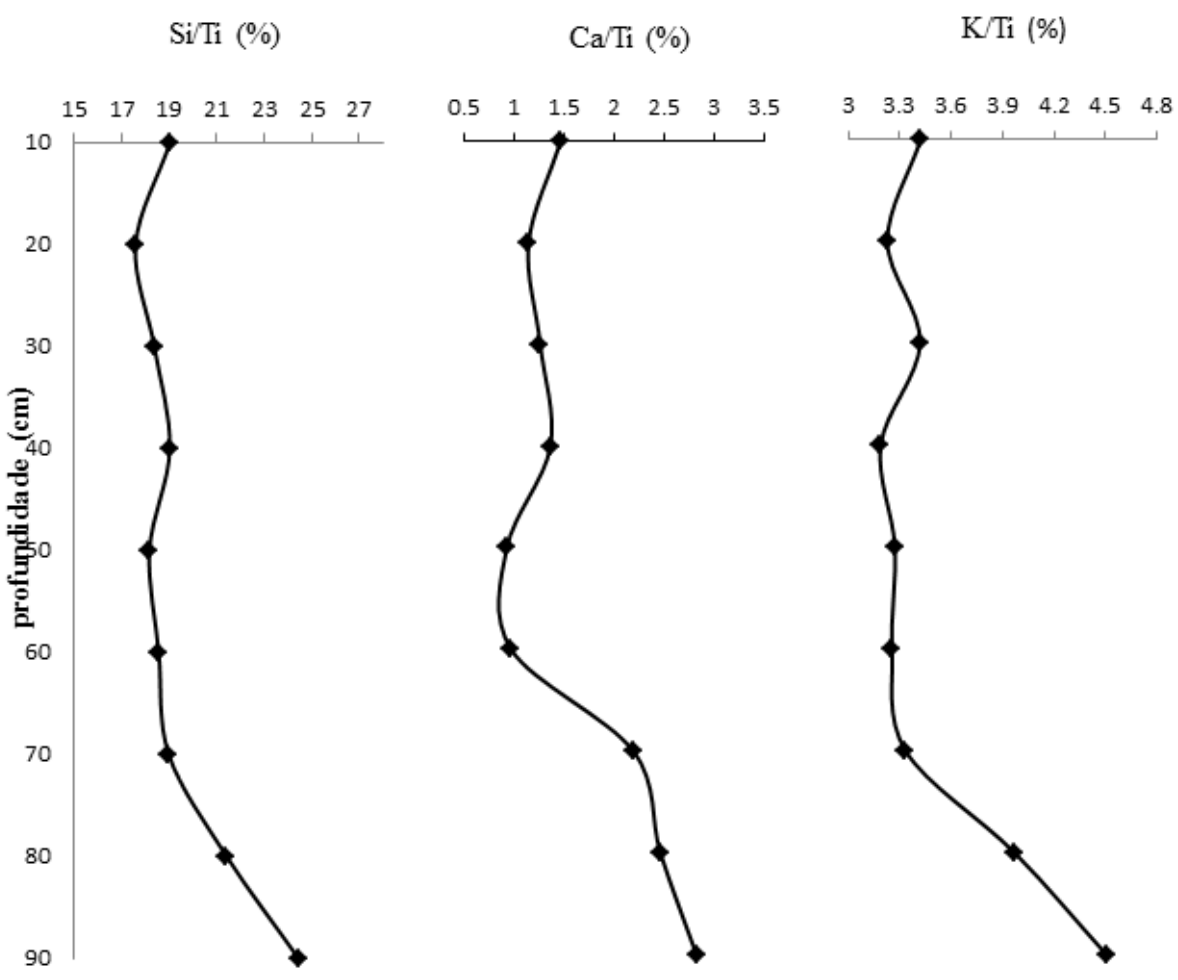

Figura 4. Perfis verticais dos indicadores da paleoprodutividade usando as razões ( $\mathrm{Si} / \mathrm{Ti}, \mathrm{Ca} / \mathrm{Ti}$ e K/Ti) ao longo da profundidade. Fonte: Langa et al. (2019).
Os três gráficos apresentam perfis com o mesmo comportamento da variação da paleoprodutividade aos $20 \mathrm{~cm}$ de profundidade nota-se um pequeno declínio, de $50 \mathrm{~cm}$ a $60 \mathrm{~cm}$ de profundidade não verifica-se variação significativa e um aumento gradual verificado dos $70 \mathrm{~cm}$ até 90 $\mathrm{cm}$, onde atinge o pico máximo. 
Na Tabela 1 são apresentados os valores do coeficiente de Pearson entre os parâmetros representativos da contribuição continental $(\mathrm{Al} / \mathrm{Ca}$, $\mathrm{Ti} / \mathrm{Ca}$ e $\mathrm{Fe} / \mathrm{Ca}$ ) e da paleoprodutividade $(\mathrm{Si} / \mathrm{Ti}$,
$\mathrm{Ca} / \mathrm{Ti}$ e K/Ti). O coeficiente de Pearson estabelece o grau de correlação entre as variáveis independentes.

Tabela 1. Valores da correlação de Pearson para os parâmetros utilizados na análise entre os elementos Fe/Ca (ferro/cálcio), Al/Ca (alumínio/cálcio), Ti/Ca (titânio/cálcio), Si/Ti (silício/titânio), Ca/Ti (cálcio/titânio) e K/Ti (potássio/titânio). Fonte: Langa et al. (2019).

\begin{tabular}{lllllll}
\hline Razões & $\mathbf{F e} / \mathbf{C a}$ & $\mathbf{A l} / \mathbf{C a}$ & $\mathbf{T i} / \mathbf{C a}$ & $\mathbf{S i} / \mathbf{T i}$ & $\mathbf{C a} / \mathbf{T i}$ & $\mathbf{K} / \mathbf{T i}$ \\
\hline $\mathrm{Fe} / \mathrm{Ca}$ & 1 & & & & & \\
$\mathrm{Al} / \mathrm{Ca}$ & 0.95231 & 1 & & & & \\
$\mathrm{Ti} / \mathrm{Ca}$ & 0.9287 & 0.97794 & 1 & & & \\
$\underline{\mathrm{Si} / \mathrm{Ti}}$ & $\underline{-0.83645}$ & $\underline{-0.77771}$ & $\underline{-0.75724}$ & 1 & & \\
$\underline{\mathrm{Ca} / \mathrm{Ti}}$ & $\underline{-0.96461}$ & $\underline{-0.96218}$ & $\underline{-0.96031}$ & 0.87266 & 1 & \\
$\underline{\mathrm{K} / \mathrm{Ti}}$ & $\underline{-0.84151}$ & $\underline{-0.76431}$ & $\underline{-0.72907}$ & 0.96869 & 0.85169 & 1 \\
\hline
\end{tabular}

É possível observar que existe uma relação forte negativa entre os valores dos parâmetros da contribuição continental e a paleoprodutividade.

\section{Discussão}

As razões elementares de $\mathrm{Al} / \mathrm{Ca}, \mathrm{Ti} / \mathrm{Ca}$ e $\mathrm{Fe} / \mathrm{Ca}$ têm sido usados em muitos estudos de reconstrução paleoambiental, sendo considerados indicadores promissores de contribuição continental (Pinheiro, 2013). Isto é baseado na premissa de que o Cálcio $(\mathrm{Ca})$ reflete o conteúdo carbonático marinho no sedimento, representando os componentes biogénicos. Por sua vez, o Titânio (Ti), Alumínio (Al) e o Ferro (Fe) estão relacionados aos componentes siliciclásticos ou aluminossilicatos e aos minerais essencialmente argilosos (Arz et al., 1998).

Os perfis verticais das razões elementares $\mathrm{Al} / \mathrm{Ca}, \mathrm{Ti} / \mathrm{Ca}$ e $\mathrm{Fe} / \mathrm{Ca}$ apresentam um certo padrão das flutuações das entradas continentais no estuário de Macuse. Os valores mínimos verificam-se na base do testemunho aos $90 \mathrm{~cm}$ de profundidade e os valores máximos na profundidade média aos 50 $\mathrm{cm}$.

Os valores mínimos e ou baixos das razões elementares $\mathrm{Al} / \mathrm{Ca}, \mathrm{Ti} / \mathrm{Ca}$ e $\mathrm{Fe} / \mathrm{Ca}$ são interpretados como um sinal da fraca contribuição do material terrígeno no estuário. De acordo com (Campos, 2019), valores baixos da contribuição continental pode ser associado a tempo seco, no qual há baixa intensidade da precipitação na região.

Altos valores das razões elementares $\mathrm{Al} / \mathrm{Ca}, \mathrm{Ti} / \mathrm{Ca}$ e Fe/Ca indicam forte contribuição do material continental dentro do estuário, que pode ser relacionado às condições relativamente húmidas, em que a intensificação da precipitação promove o fornecimento de sedimentos com assinatura geoquímica rica em Al, Fe e Ti (Souza, 2016).

Em todos os gráficos, aos $50 \mathrm{~cm} \mathrm{de}$ profundidade, registam-se valores máximos das razões elementares $\mathrm{Al} / \mathrm{Ca}, \mathrm{Ti} / \mathrm{Ca}$ e $\mathrm{Fe} / \mathrm{Ca}$ indicando que a precipitação tenha sido intensa e favoreceu maior intemperismo das rochas fonte desses elementos, proporcionando maior escoamento dos mesmos para dentro do estuário. Muhate (2019), no seu estudo de paleoprodutivididade na mesma área de estudo aos $50 \mathrm{~cm}$ de profundidade evidenciou uma diminuição do conteúdo de carbonato de cálcio que contribuiu provavelmente para o desaparecimento de espécies calcárias, impedidas de construírem o seu exo-esqueleto. Em adição, Shinu (2008) afirma que maior suplemento da contribuição continental influencia na diluição do conteúdo de carbonato de cálcio $\left(\mathrm{CaCO}_{3}\right)$.

Em vários estudos de paleoprodutividade, os indicadores geoquímicos que registam o fluxo de partículas na coluna de água têm sido utilizados como indicadores para a produtividade, assumindo-se que a maior parte do material particulado que afunda na água é de origem biogênica. Nesta abordagem, pressupõe-se que mudanças na entrega de $\mathrm{Si}, \mathrm{Ca}$ e $\mathrm{K}$ são proporcionais ao fluxo de partículas que soterram, que pode ser convertido em produtividade, portanto o uso das razões elementares $(\mathrm{Si} / \mathrm{Ti}, \mathrm{Ca} / \mathrm{Ti}$ e K/Ti) são aplicadas como indicadores de produtividade, desde que o $\mathrm{Ti}$ seja, exclusivamente, de origem terrígena (François, 2008).

Nas profundidades de $50 \mathrm{~cm} \mathrm{e} 60 \mathrm{~cm}$ em todos os perfis não se verifica flutuações significativas dos indicadores de produtividade ( $\mathrm{Si} / \mathrm{Ti}, \mathrm{Ca} / \mathrm{Ti}$ e $\mathrm{K} / \mathrm{Ti}$ ) e os seus valores são mínimos, o que indica eventos de baixa produtividade no estuário de Macuse.

Entre as profundidades $70 \mathrm{~cm}$ a $90 \mathrm{~cm}$ há registo de alta taxa de paleoprodutividade evidenciada por altos valores das razões elementares ( $\mathrm{Si} / \mathrm{Ti}, \mathrm{Ca} / \mathrm{Ti}$ e K/Ti). Para Andrade (2008), eventos de alta produtividade estão 
relacionados ao aumento do fluxo de partículas no ambiente estuarino.

Paytan (2006) acrescenta que uma das principais fontes de acumulação do $\mathrm{Ca}, \mathrm{K}$ e Si são os organismos betónicos, tais como: moluscos, briozoários, algas e equinodermes, incorporados nas suas partes duras quando morrem fazem parte da composição dos sedimentos.

No cenário atual de mudanças climáticas globais, reconstruções passadas de paleoprodutividade são indispensáveis. Muitos casos de estudo de reconstrução paleoambiental, com maior enfoque na paleoprodutividade, há que considerar a relação entre a contribuição continental e sua influência na paleoprodutividade. Isto é baseado no princípio de que a entrada de materiais continentais para o ambiente marinho estimula as condições de produtividade dentro do ambiente através do suplemento de nutrientes (Mahiques, 1998).

Os perfis verticais de paleoprodutividade ( $\mathrm{Si} / \mathrm{Ti}, \mathrm{Ca} / \mathrm{Ti}$ e $\mathrm{K} / \mathrm{Ti}$ ) apresentam valores elevados nas profundidades de $10 \mathrm{~cm}, 70 \mathrm{~cm}$ a $90 \mathrm{~cm}$ mostrando uma relação inversa com os indicadores de contribuições continentais $(\mathrm{Al} / \mathrm{Ca}, \mathrm{Ti} / \mathrm{Ca}$ e $\mathrm{Fe} / \mathrm{Ca}$ ), que apresentam valores baixos a estas profundidades. A relação inversa entre a paleoprodutividade e as contribuições continentais é realçada pelas correlações fortes negativas entre os parâmetros, como demonstrado na Tabela 1.

A relação entre a paleoprodutividade e as contribuições continentais no estuário de Macuse é contraditória se levar-se em conta o princípio de que a produtividade é estimulada pelo aporte continental. A contradição pode estar associada à capacidade de retenção de indicadores de contribuição continental pelos sedimentos durante os períodos húmidos e produtivos. Durante os períodos húmidos, geralmente caracterizados por maior hidrodinâmica e escoamentos continentais, espera-se maior presença de sedimentos grosseiros que tendem a apresentar menor adsorção, sendo que, apesar de maior entrada de indicadores continentais, esses não são retidos em sedimentos. Esta possibilidade é reforçada por Schaefer (2015), ao afirmar que os elementos tendem a ser lixiviados da fração arenosa dos sedimentos (que possui baixa relação área/volume) por meio da percolação de fluidos.

$$
\text { Muhate (2019) estudou a }
$$

paleoprodutividade da mesma área de estudo usando a matéria orgânica como indicador, tendo encontrado resultados contrários aos do presente estudo, visto que observou baixos valores de matéria orgânica nas profundidades de $70 \mathrm{~cm}$ a 90 $\mathrm{cm}$.
Os baixos valores da matéria orgânica encontrados por Muhate (2019), nas profundidades $70 \mathrm{~cm}$ a $90 \mathrm{~cm}$, podem estar associados a concentrações de areia que são relativamente altas, se comparadas a outras profundidades.

A matéria orgânica (MO) é adsorvida da fração fina de sedimentos (silte), pois apresentam maior coesão entre as partículas, atuando como um cimentador e apresentam uma grande área de superfície em relação à fração arenosa (areia) (Andrade, 2011).

\section{Conclusão}

As flutuações da paleoprodutividade apresentam uma relação inversa com os indicadores das contribuições (entradas) continentais, sugerindo que a paleoprodutividade no estuário de Macuse não foi estimulada pelo aporte continental;

Os eventos de alta produtividade coincidiram com altos valores de areia, identificados como sendo de tempo húmido com forte hidrodinâmica e escoamentos continentais. As contribuições continentais apresentam valores baixos nesses eventos, podendo relacionar estas incoerências à fraca capacidade de retenção que a areia apresenta para com os indicadores da contribuição continental.

\section{Agradecimentos}

Os autores agradecem ao laboratório de química da Faculdade de Ciências da Universidade Eduardo Mondlane pela realização das análises.

\section{Referências}

Andrade, M. M. 2008. Paleoprodutividade costeira da região de Cabo Frio, Rio de Janeiro, ao longo dos últimos 13.000 anos cal AP. Tese de Doutoramento, Universidade Federal Fluminense, Departamento de Geoquimica, Niteró, 36p.

Andrade, P. C. 2011. Varições de paleoprodutividade na plataforma continental interna ao largo de Itajai-SC durante o Holoceno: uma abordagem de multiindicadores. Dissertação de Mestrado, Universidade São Paulo, São Paulo, 6p.

Arz, H. W.; Jurgen, P. G. 1998. Correlated Millennial-Scale Changes in Surface Hydrography and errigenous Sediment Yield Inferred from Last-Glacial Marine Deposits off Northeastern Brazil. Quaternary research, pp. 157-166.

Blanchet, C. L.; Thouveny, N.; Vidal, L.; Leduc, G. 2007. Terrigenous input response to glacial/interglacial climatic variations over southern Baja California: a rock magnetic 
approach. Elsevier, 3121p. http://dx.doi.org/10.1016/j.quascirev.2007.0 7.008

Campos, M. S. 2019. Padrões hidrodinâmicos no atlântico sudoeste durante o holoceno: uma abordagem multi-proxy. Dissertação de Mestrado, Universidade Federal do Paraná, Sistemas Costeiros e oceanicos, Pontal do Parana, 12p.

Francois, R. 2008. Ocean paleoproductivity. pp. 643-651.

Lamb, A. L.; Wilson, G. P.; Leng, M. J. 2006. A review of coastal palaeoclimate and relative sea-level reconstructions using $13 \mathrm{C}$ and $\mathrm{C} / \mathrm{N}$ ratios in organic material. Elsevier, pp. 29-57. http://dx.doi.org/10.1016/j.earscirev.2005.10 .003

Mahiques, M. M. 1998. Caracteristicas da materia orgânica sedimentar da plataforma continetal interna e média entre a Baia da Guanabara (RJ) e São Francisco do Sul (SC). Tese de Doutoramento, Unversidade de São Paulo, Instituto Oceanografico, São Paulo, 86p.

Martins, V.; Jouanneau, J.; Weber, O.; Rocha, F. 2006. Tracing the late Holocene evolution of the NW Iberian upwelling system. Marine Micropaleontology, 59, 35-55. http://dx.doi.org/10.1016/j.marmicro.2005.1 2.002

Martins, V.; Dubert, J.; Joauneau, J.; Weber, O.; Patinha, C.; E.; F. S.; et al. 2007. A multiproxy aproach of the Holocene evolution of shelf-slop circulation of the NW Iberian Continental shelf. Marine Geology, 239, $1-18$. http://dx.doi.org/10.1016\%2Fj.margeo.2006. 11.001

Miguel, L. L.; Nehama, F. P.; Castro, J. W. 2017. A Mecânica do Transporte de Sedimentos em Suspensão no Estuário do Rio Macuse, Moçambique, Sudeste de África. Revista Brasileira de Geomorfologia, 18, (1), 107123.

http://dx.doi.org/10.20502/rbg.v18i1.830

Muhate, A. C. 2019. Estudo da Paleoprodutividade do Estuário de Macuse durante o Holoceno. Monografia, Universidade Eduardo Mondlane, Geologia Marinha, Quelimane, 43p.

Nagai, R. H. 2009. Variações de paleoprodutividade na plataforma interna de Cabo Frio, RJ, durante o Holoceno.
Universidade de São Paulo, Instituto Oceanográfico, Universidade de São Paulo, São Paulo, 130p.

Paytan, A. 2006. Ocean Paleoproductivity. Encyclopedia of Paleoclimatology and Ancient Environments. In: Gornitz, V. (Ed). Encyclopedia of Earth Science Series. Kluwer Academic Publishers. pp. 4453-4455.

Piñeiro, N. L. 2013. Determinação do grau da influência terrestre versus marinha ao longo da transição Rio de La Plata - Oceano Atlântico Sul, através de proxies bióticos e abióticos. Dissertação de Mestrado, Universidade de São Paulo, Instituto Oceanográfico, Sao Paulo, 51p.

Ramos, R. D. 2010. Paleoceanografia do sudoeste do Atlântico Sul: Registo de eventos abruptos nos últimos 50.000 anos. Tese de Doutoramento, Instituto de Quimica, Universidade Federal de Fluminense, Niterói, $133 \mathrm{p}$.

Santos, C. T. 2010. Reconstrução das condições paleoambientais e paleoclimáticas no estuário do Tejo durante o Holocénico ( 12000 anos). Tese Doutorado, Universiadde do Porto, Departamento de Mestrado em Ciencias do Mar, Porto, $147 \mathrm{p}$.

Schaefer, G. L. 2015. Retenção e liberação de fósforo em sedimento de fundo de açudes e córregos de pequenas bacias hidrográficas em um assentamento de reforma agrária. Dissertacao de Mestrado, Universidade Federal de Santa Maria, Centro de Ciencias Socias, Santa Maria, RS, Brasil, 88p.

Shinu, N. 2008. Environment and Climate Changes during the Late Quaternary: Inferences from Sedimentary Records of Southeastern Arabian Sea. Tese de Doutoramento, Cochin University of Science and Technology, Department of Marine Geology, Geophysics, $155 \mathrm{p}$.

Souza, A. L. 2016. Variações no aporte terrígeno para o mar sul da china nos últimos $400 \mathrm{mil}$ anos a partir de razões elementares de um testemunho marinho. Dissertação, Unversidade Federal do Paraná, Ciências da Terra, Pontal do Paranà, Brazil, 50p.

Veronez Junior, P. 2009. Ecofácies e faciologia dos sedimentos de fundo da baía de Vitória - E.S. Dissertação de Mestrado, Universidade Federal do Espírito Santo, Departamento de oceanografia e ecologia, Vitória, 118p. 